\title{
VARIACIÓN ESTACIONAL DE SAPONINAS EN SOLIDAGO CHILENSIS VAR. CHILENSIS (ASTERACEAE)
}

\author{
ANA M. ARAMBARRI ${ }^{1,2^{*}}$ y MARCELO P. HERNÁNDEZ $Z^{1,3}$
}

\begin{abstract}
Summary: Seasonal variation of saponins in organs of Solidago chilensis var. chilensis (Asteraceae). Solidago chilensis Meyen var. chilensis is a widely distributed weed in Argentina. Owing to the presence of saponins, S. chilensis is a poisonous weed for cattle. However, it has several medicinal uses and it is considered a melliferous species. The purpose of this study was to analyze the seasonal variability in the saponin content of subterranean and aerial organs of S. chilensis by a qualitative method. Using an aqueous solution obtained from fresh plant material of different organs (rhizomes, roots, stems, leaves and inflorescences), the saponin content was determined by the height of the foam column of the solution. Saponins were always present in subterranean organs of the plant (roots and rhizomes), but also gave a highly positive reaction in leaves from the end of summer to early autumn (March-April). Accordingly, between March and April, is the most suitable period to extract saponins from S. chilensis under natural conditions.
\end{abstract}

Key words: Aerial organs, cattle, medicinal plant, poisonous weed, subterranean organs.

Resumen: Solidago chilensis Meyen var. chilensis es una maleza ampliamente distribuida en Argentina. $S$. chilensis es una especie tóxica para el ganado debido a la presencia de saponinas. Sin embargo, tiene diversos usos medicinales y es además una especie melífera. El objetivo de este estudio fue analizar la variabilidad estacional en el contenido de saponinas presentes en órganos subterráneos y aéreos de $S$. chilensis, por medio de un método cualitativo. El contenido de saponinas se determinó por la altura de la columna de espuma de una solución acuosa obtenida a partir de material vegetal fresco de diferentes órganos de la planta (rizomas, raíces, tallos, hojas e inflorescencias). Los órganos subterráneos presentaron saponinas durante todo el año, pero la presencia en hojas también resultó altamente positiva en verano y otoño (marzo-abril). Estos resultados sugieren que esta última sería la época del año apropiada para extraer saponinas de $S$. chilensis en condiciones naturales.

Palabras clave: Ganado, maleza tóxica, órganos aéreos, órganos subterráneos, planta medicinal.

\section{INTRODUCCIÓN}

Solidago chilensis Meyen var. chilensis (Asteraceae), conocida como "cola de zorro" o "falso romerillo" entre otros (De la Peña \& Pensiero, 2004), es una hierba perenne rizomatosa con ciclo estival (primavera-verano-otoño) y maleza invasora

${ }^{1}$ Laboratorio de Morfología Comparada de Espermatófitas (LAMCE).

${ }^{2}$ Cátedra de Morfología Vegetal. E-mail: anaramba@ yahoo.com.ar

${ }^{3}$ Cátedra de Sistemática Vegetal, Facultad de Ciencias Agrarias y Forestales, Cátedra de Botánica Sistemática II, Facultad de Ciencias Naturales y Museo, Universidad Nacional de La Plata. E-mail: mphciencia@yahoo.com difícil de extirpar (Boelcke, 1981; Marzocca et al., 1986) (Fig. 1). Habita en Argentina, Bolivia, Brasil, Chile, Paraguay y Uruguay. En nuestro país, posee una amplia distribución, creciendo desde el nivel del mar hasta 2500 m s.n.m. (Cabrera, 1963; Cabrera \& Burkart, 1974; Zuloaga et al., 2008). S. chilensis es una especie melífera y se ensaya como ornamental para regiones semiáridas (Gil et al., 2012). Se destaca su aprovechamiento medicinal como anticefalálgico, antihelmíntico, antidiurético, antioxidante, antilítico, antirrehumático, antiséptico, antiinflamatorio y gastroprotector (Güntner et al., 1999; Freire \& Urtubey, 2000; Carrizo et al., 2002; Lahitte et al., 2004; Rodríguez et al., 2004; Pensiero et al., 2005; Barboza et al., 2006; Bucciarelli et al., 2007; Zampini et al., 2007). En uso externo se 


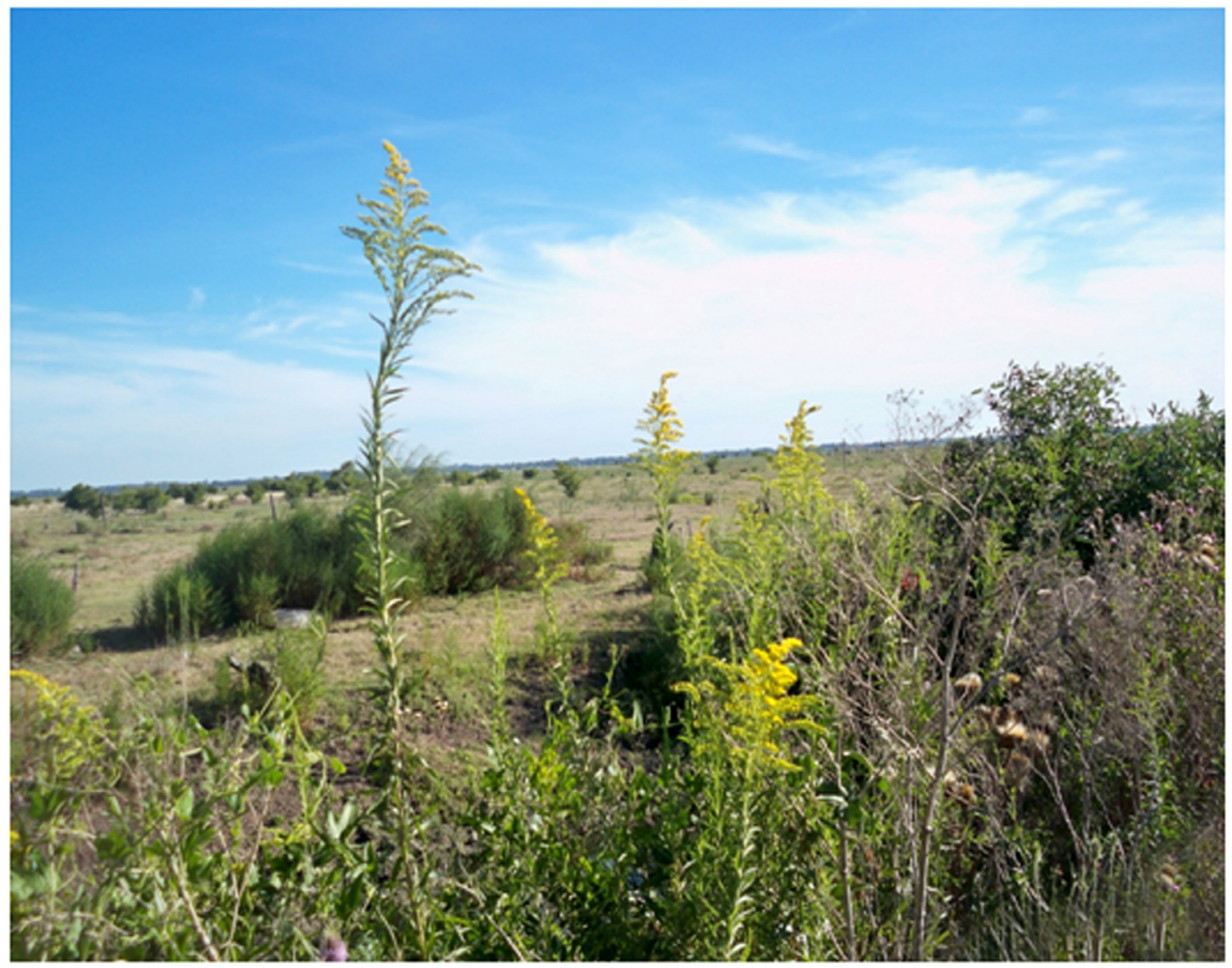

Fig. 1. Solidago chilensis var. chilensis. Avenida Río de La Plata, Pdo. Berisso, Prov. Buenos Aires, Argentina.

emplea para traumas y contusiones (Valverde et al., 2011). Entre los compuestos químicos se han hallado aceites esenciales, almidón, compuestos del grupo lactónico, flavonoides, heterósidos antraquinónicos, resinas, saponinas y taninos (Domínguez, 1928; Hernández et al., 2012, 2013a). Las saponinas serían los compuestos responsables de su toxicidad para el ganado (Gallo, 1979), pero también tienen propiedades biológicas y farmacológicas (Sparg et al., 2004); y son utilizadas como agente fungicida en patologías humanas, animales y vegetales (Gattuso, 1996; Escalante et al., 2002; Zacchino, 2004; Hernández et al., 2013b).

El contenido de metabolitos secundarios está influenciado por el genotipo de la planta (especie, variedad) y las características ambientales como radiación solar y disponibilidad de agua, la velocidad de crecimiento, la madurez, la condición nutricional del suelo, la depredación y las enfermedades (Waterman \& Mole, 1994). Así mismo, la aparición de los compuestos secundarios está relacionada con los mecanismos de defensa de la planta y los efectos del suelo y del clima (Harborne, 1993). Según plantea Kumar (1997), los distintos compuestos que puede producir una especie presentan una determinada distribución dentro de los órganos, los tejidos y las células de una planta, y ello responde a menudo a las influencias ambientales. La amplia distribución de $S$. chilensis var. chilensis, fácil reproducción, abundancia y contenido en saponinas con potencial aplicación en el control fúngico, justifican la elección de la especie para comenzar los estudios. El propósito fue establecer el órgano vegetal y la época del año más favorable para la extracción 
de saponinas y su posterior ensayo en control biológico. Sobre esa base para el presente trabajo se fijaron los siguientes objetivos: (1) determinar el contenido de saponinas en los diferentes órganos, subterráneos (raíz-rizoma) y aéreos (tallo, hoja e inflorescencia); (2) establecer la variación del contenido de saponinas en cada órgano en las diferentes estaciones del año.

\section{Material y Método}

El material de plantas completas fue recolectado a orillas de la avenida Río de La Plata (3450'49,40" S y $57^{\circ} 50^{\prime} 48,49^{\prime \prime}$ O), en los talares del Partido de
Berisso, entre las ciudades de La Plata y Berisso, provincia de Buenos Aires, Argentina. Ejemplares de referencia se encuentran depositados en el herbario de la Facultad de Agronomía de La Plata (Hernández 99-103, LPAG). Los ensayos se realizaron en enero-febrero-marzo (verano); abril y mayo (otoño), julio-agosto (invierno) y octubrenoviembre (primavera). El material se recolectó al atardecer, se lavó para retirar las partículas de suelo, se fraccionó y tomaron fotografías de planta entera y órganos fraccionados (Fig. 2). A las 24 horas se determinaron las saponinas. Para la determinación de saponinas se pesaron $10 \mathrm{~g}$ de cada órgano (raízrizoma; tallo; hoja; inflorescencia) (Fig. 2). En cada caso se molió la muestra en $100 \mathrm{~mL}$ de agua

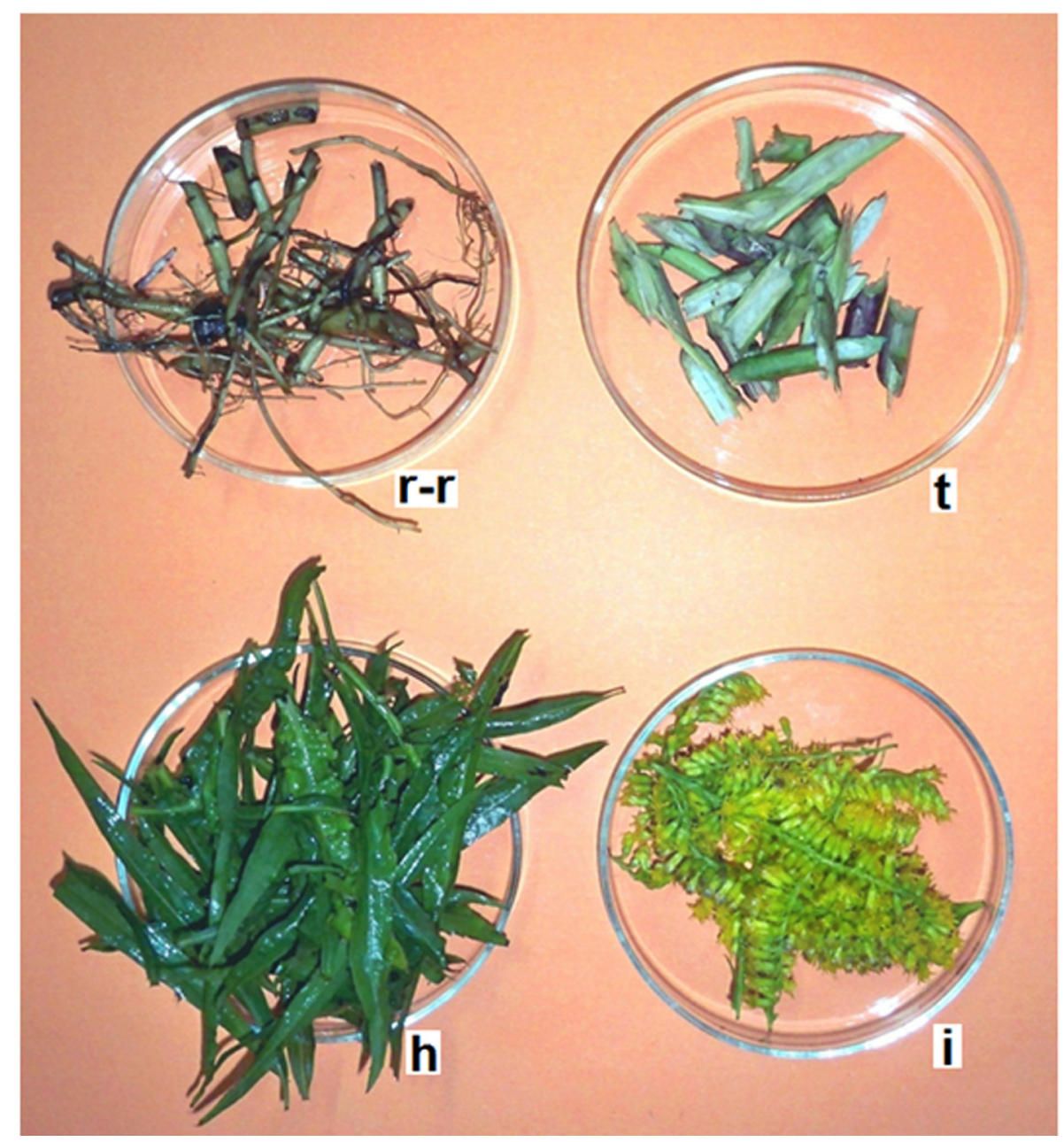

Fig. 2. Órganos de Solidago chilensis var. chilensis fraccionados. Ref. r-r: raíz-rizoma; t: tallo; h: hoja; i: inflorescencia. 
destilada. El producto triturado se hirvió a baño María durante $10 \mathrm{~min}$. Una vez frio se filtró y el líquido de filtrado se enrasó a $100 \mathrm{~mL}$ con agua destilada. Para cada uno de los órganos, de los 100 $\mathrm{mL}$ preparados, se tomaron alícuotas crecientes de 1 a $10 \mathrm{~mL}$ y vertieron en diez tubos de ensayo (Tabla 1).

Una vez preparadas las diluciones, los tubos se agitaron en sentido vertical durante $15 \mathrm{~s}$, después de 15 min se observó la persistencia de la columna de espuma; para evaluar el contenido de saponinas se utilizó la escala de Harborne (1989) que establece de acuerdo a la altura de la columna de espuma: menor a $1 \mathrm{~cm}$, prueba ligeramente

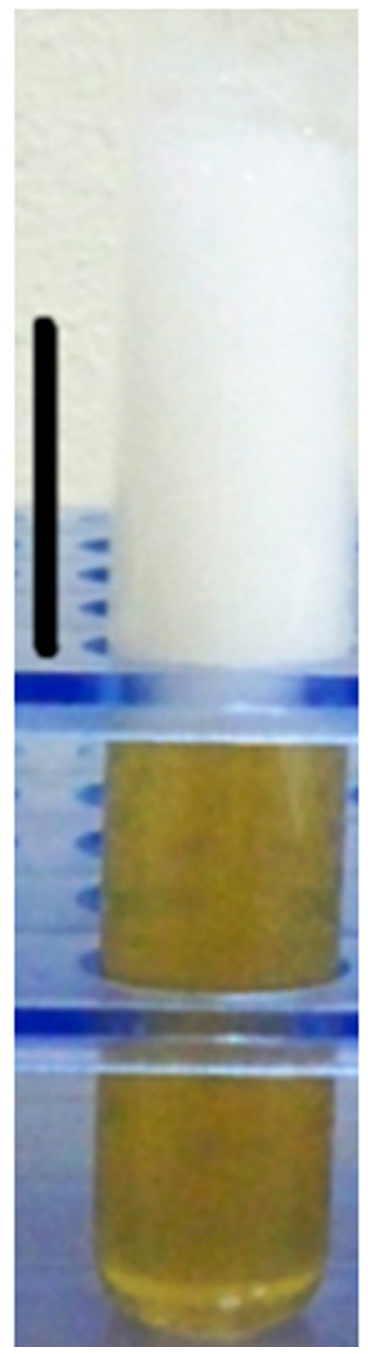

Fig. 3. Columna de espuma de la hoja en marzo. Escala: $2 \mathrm{~cm}$. positiva; igual a $2 \mathrm{~cm}$, prueba positiva; y mayor a 2 $\mathrm{cm}$, prueba fuertemente positiva.

Los datos meteorológicos fueron proporcionados por el personal del Departamento de Sismología e Información Meteorológica, Facultad de Ciencias Astronómicas y Geofísicas, Universidad Nacional de La Plata (Tabla 2).

Tabla 1. Dilución del extracto acuoso. TE: tubos
de ensayo; EA: extracto acuoso; AD: agua
destilada (mL).
\begin{tabular}{|cccccccccccc|}
\hline TE & I & II & III & IV & V & VI & VII & VIII & IX & X \\
\hline EA (mL) & 1 & 2 & 3 & 4 & 5 & 6 & 7 & 8 & 9 & 10 \\
AD (mL) & 9 & 8 & 7 & 6 & 5 & 4 & 3 & 2 & 1 & 0 \\
\hline
\end{tabular}

Tabla 2. Valores medios mensuales de Temperatura $\left({ }^{\circ} \mathrm{C}\right)$ y Lluvias $(\mathrm{mm})$, año 2012.

\begin{tabular}{|lcc|}
\hline \multicolumn{1}{|c}{ Mes } & Temperatura $\left({ }^{\circ} \mathrm{C}\right)$ & Lluvias $(\mathrm{mm})$ \\
\hline ENE & 24,2 & 39,2 \\
FEB & 23,6 & 170,2 \\
MAR & 20,5 & 69,8 \\
ABR & 16,5 & 38,2 \\
MAY & 15,3 & 73,6 \\
JUN & 10,4 & 21,4 \\
JUL & 7,9 & 26,4 \\
AGO & 12,3 & 209 \\
SEP & 14,1 & 48,6 \\
OCT & 16,9 & 182,4 \\
NOV & 20,8 & 57 \\
DIC & 23,2 & 183,8 \\
\hline
\end{tabular}

\section{Resultados}

De todos los órganos analizados, los subterráneos (raíz-rizoma) tuvieron contenido de saponinas durante todo el año, con valores elevados en invierno que se mantuvieron hasta mediados de primavera; los tallos, presentes en primavera, verano y otoño mostraron un bajo contenido de saponinas en todas las estaciones, alcanzando un máximo de $1 \mathrm{~cm}$ de columna de espuma en primavera. Las hojas si bien contienen saponinas en primavera y verano sólo registraron un resultado fuertemente positivo hacia fines de verano, comienzo de otoño (marzo-abril) (Fig. 3). Las inflorescencias presentes en el período estivo-otoñal 
Tabla 3. La variación estacional del contenido de saponinas en los órganos de S. chilensis var. chilensis se expresa de acuerdo a la escala de Harborne (1989). Ref.: prueba negativa o ausencia del órgano en la estación del año.

\begin{tabular}{|lllll|}
\hline \multicolumn{1}{|c}{$\begin{array}{c}\text { Estaciones } \\
\text { del año }\end{array}$} & \multicolumn{1}{c}{$\begin{array}{c}\text { Raíz-rizoma } \\
(\mathbf{r}-\mathbf{r})\end{array}$} & \multicolumn{1}{c}{ Tallo } & \multicolumn{1}{c|}{ Hoja } & \multicolumn{1}{c|}{ Inflorescencia } \\
Verano & positiva & ligeramente positiva & ligeramente positiva & negativa \\
Otoño & positiva & ligeramente positiva & fuertemente positiva & ligeramente positiva \\
Invierno & fuertemente positiva & negativa & negativa & negativa \\
Primavera & fuertemente positiva a positiva & ligeramente positiva & positiva & negativa \\
\hline
\end{tabular}

mostraron escaso contenido de saponinas, formando una columna de espuma entre 1 y 4 mm (Tabla 3). En la Fig. 4 se indican los valores medios de temperatura y lluvias durante el año de ensayo.

\section{Discusión}

Las saponinas son un compuesto tóxico para el ganado y las inflorescencias serían el órgano con mayor contenido (Gallo, 1979). De acuerdo con

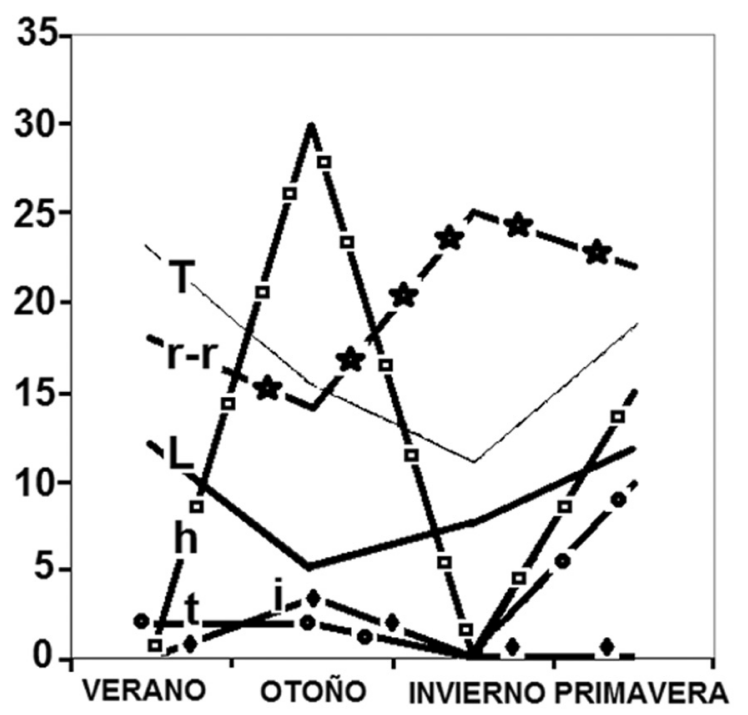

Fig. 4. Variación del contenido de saponinas en los diferentes órganos en cada estación con las condiciones de temperatura y lluvia dadas. Eje $x$, representa los $\mathrm{mm}$ alcanzados por la columna de espuma para cada órgano. Las estaciones del año

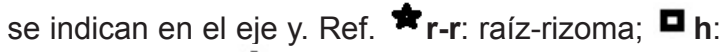
hoja; t: tallo; ${ }_{\mathrm{i}}$ : inflorescencia. T: Temperatura media mensual en grados centígrados y L: Lluvia media mensual expresada en centímetros. nuestras determinaciones en las inflorescencias de S. chilensis hay un bajo contenido de saponinas. La floración de esta especie, sin embargo, coincide con las hojas desarrolladas y un elevado contenido de saponinas en las mismas, por lo que serían las hojas consumidas por el ganado resultan tóxicas. Por otra parte, el alto contenido de saponinas en este órgano resulta promisorio para su extracción y ensayo en control biológico, sobre la base de los antecedentes brindados por varios autores (Moreno \& Rodríguez, 1981; Escalante, 2002; Farías Magalhaes et al., 2003; Zacchino, 2004; Hernández et al., 2012). Al respecto, la actividad de las saponinas en el control de hongos patógenos se basaría en la capacidad de formar complejos con los esteroles de la membrana produciendo su desintegración (Montes-Belmont, 2009).

\section{Conclusión}

Solidago chilensis resulta una especie promisoria como productora de saponinas dado el elevado contenido existente en sus órganos. La hoja recolectada en marzo-abril es el órgano sugerido para la extracción de saponinas en el área indicada y con las condiciones climáticas consideradas. Se han sentado las bases para ensayar la posible acción de las saponinas sobre hongos fitopatógenos.

\section{Agradecimientos}

Al personal del Departamento de Sismología e Información Meteorológica de la Facultad de Ciencias Astronómicas y Geofísicas, UNLP por proveernos los registros climáticos. Lo hacemos extensivo a María Alejandra Migoya por su colaboración en la preparación de las Figuras. 


\section{Biblografía}

BARBOZA, G., J. J. CANTERO, C. O. NÚÑEZ \& L. ARIZA ESPINAR. 2006. Flora medicinal de la provincia de Córdoba (Argentina), Museo Botánico de Córdoba, Gráficamente ediciones, Córdoba.

BOELCKE, O. 1981. Plantas vasculares de la Argentina, nativas y exóticas. Fundación para la Educación, la Ciencia y la Cultura, Buenos Aires.

BUCCIARELLI, A., M. M. MANCINI \& M. I. SKLIAR. 2007. Estudios de la actividad gastroprotectora de Solidago chilensis. Bol. Latinoam. Caribe 6: 332-333.

CABRERA, A. L. (ed.). 1963. Compuestas. En: Flora de la provincia de Buenos Aires. Colecc. Ci. Inst. Nac. Tecnol. Agropecu. 4: 443.

CABRERA, A. L. \& A. BURKART. 1974. Compuestas. En: BURKART A. (ed.) Flora ilustrada de Entre Ríos 6: 106-554. Colecc. Ci. Inst. Nac. Tecnol. Agropecu., Buenos Aires.

CARRIZO, E., M. O. PALACIO \& L. D. ROIC. 2002. Plantas de uso medicinal en la flora de los alrededores de la ciudad de Santiago del Estero (Argentina). Dominguezia 18: 26-35.

DE LA PEÑA, M. R. \& J. F. PENSIERO. 2004. Plantas argentinas. Catálogo de nombres comunes. Ed. L.O.L.A., Buenos Aires.

DOMÍNGUEZ, J. A. 1928. Contribuciones a la materia médica Argentina. Instituto de Botánica y Farmacología (Facultad de Ciencias Médicas de Buenos Aires) Ed. Peuser, Buenos Aires.

ESCALANTE A. M., C. B. SANTECCHIA, S. N. LÓPEZ, M. A. GATTUSO, A. GUTIÉRREZ, F. DELLE MONACHE, M. GONZÁLEZ SIERRA \& S. A. ZACCHINO. 2002. Isolation of antifungal saponins from Phytolacca tetramera, an Argentinean species in critic risk. J Ethnopharmacol. 1: 29-34.

FARIAS MAGALHÃES, A., A. M. GOULART DE AZEVEDO TOZZI, C. CAPARICA SANTOS, D. R. SERRANO, E. M. ZANOTTI-MAGALHÃES, E. GONÇALVES MAGALHÃES \& L. A. MAGALHÃES. 2003. Saponins from Swartzia langsdorffii: biological activities. Mem. Inst. Oswaldo Cruz, Río de Janeiro 98: 713-718.

FREIRE, S.E. \& E. URTUBEY. 2000. Compuestas medicinales de la provincia biogeográfica pampeana. Claves para su determinación e iconografías. Parte 5: (Grupos 6 y 7). Acta Farmacéutica Bonaerense 19: 165-184.

GALLO, G. G. 1979. Plantas tóxicas para el ganado en el cono sur de América. Ed. Universitaria de Buenos Aires.

GATTUSO M.A. 1996. Estudio anatómico, ultraestructural y fitoquímico de las Phytolaccaceae de la Argentina. Tesis Doctoral, Facultad de Ciencias Bioquímicas y Farmacéuticas, Universidad Nacional de Rosario.
GIL, S. P., L. SEISDEDOS, M. E. REYNA \& M. M. CERANA. 2012. Epidermis foliar de tres especies de asteráceas nativas de Argentina con potencial ornamental. Int. J. Exp. Bot., ФHYTON 81: 205-210.

GÜNTNER, C., C. BARRA, M. V. CESIO, E. DELLACASSA, L. FERRANDO, F. FERREIRA, C. GARCÍA, G. GONZÁlEZ, H. HEINZEN, A. LLORET, D. LORENZO, P. MENÉNDEZ, D. PAZ, S. SOULE, A. VÁZQUEZ \& P. MOYNA. 1999. Antioxidant properties of Solidago chilensis L. flavonoids. Acta Hort. 501: 159-163.

HARBORNE, J.B. 1989. Phytochemical methods: a guide to modern techniques of plant analysis. 3ra. ed. Chapman and Hall, New York.

HARBORNE, J.B. 1993. Introduction to ecological biochemistry, 4th ed., Academic Press, London.

HERNÁNDEZ M. P., A. M. ARAMBARRI, S. M. MARTÍNEZ ALONSO \& L. A. MORANDI. 2012. Morfo-histología, técnicas histológicas y ensayos fitoquímicos preliminares en Solidago chilensis (Asteraceae). XIV Jornadas Fitosanitarias Argentinas. Acta de resúmenes, Sección malezas, pág. 175. UNSL/ FICES.

HERNÁNDEZ M. P., S. M. MARTÍNEZ ALONSO, L. A. MORANDI \& A. M. ARAMBARRI. 2013a. Anatomical and chemical analysis in Solidago chilensis var. chilensis (Asteraceae). Lat. Am. J. Pharm. 32: 1236-1240.

HERNÁNDEZ M. P., M. MURACE, J. RINGUELET, I. PETRI, D. GALLO \& A. M. ARAMBARRI. 2013b. Effect of aqueous and alcohol extracts of the Phytolacca tetrámera (Phytolaccaceae) leaves on Colletotrichum gloeosporioides (Ascomycota). Bol. Soc. Argent. Bot. 48: 201-209.

KUMAR, R. 1997. Anti- nutritional factors. The potential risks of toxicity and the methods to alleviate them. In: SPEEDY A. \& P. PUGLIESE (Eds.) Legumes trees and other fodder trees as protein sourcefor livestock. Food and Agriculture organization of the United Nations (FAO), Roma.

LAHITTE, H. B., J. A. HURRELL, M. J. BELGRANO, L. S. JANKOWSKI, P. HALOUA \& K. MEHLTRETER. 2004. Plantas medicinales rioplatenses. Ed. L.O.L.A., Buenos Aires.

MARZOCCA, A., O. J. MÁRSICO \& O. DEL PUERTO. 1986. Manual de malezas. Ed. Hemisferio Sur, Buenos Aires

MONTES-BELMONT, R. 2009. Diversidad de compuestos químicos producidos por las plantas contra hongos fitopatógenos. Rev. Mex. Micol. 29: 73-82.

MORENO, M. \& V. M. RODRIGUEZ. 1981. Yiamoloside $\mathrm{B}$, a fungistatic saponin of Phytolacca octandra. Phytochemistry 20: 1446-1447.

PENSIERO J. J., J. D. MUÑOZ \& V. MARTINEZ. 2005. Proyecto Bosques Nativos y Áreas Protegidas. 


\section{A. M. Arambarri y M. P. Hernández - Saponinas en Solidago chilensis var. chilensis}

Argentina Banco Mundial - $\mathrm{N}^{\circ}$ 4085-AR. Proyectos de Investigación Aplicada los Recursos Forestales Nativos (PIARFON). Alternativas de sustentabilidad del bosque nativo del Espinal. Área Etnobotánica 45 pp. Disponible en: http://www-ambiente.gov. ar/.../web/.../PIARFON\%20MyE/Etnobotanica.pd... [Acceso: junio 2012].

RODRIGUEZ, J. A., C. BUSTAMANTE, L. ASTUDILLO \& G. SCHMEDA-HIRSMANN. 2004. Gastroprotective activity of solidagenone on experimentally-induced gastric lesions in rats. $J$. Pharm. Pharmoacol. 54: 399-402.

SPARG, S. G., M. E. LIGHAT, J. VAN STADEN. 2004. Biological activities and distribution of plant saponins. J. Ethnopharmacol. 94: 219-243.

VALVERDE S. S., S. P. DE SOUZA, K. DOS S. COPLE LIMA, T. B. OLIVEIRA \& A. L. DOS SANTOS LIMA. 2011. Selective-ion monitoring in unequivocal identification of solidagenone in Solidago chilensis raw extracts. Sociedade Brasileira de Espectrometria de Massas, $4^{\circ}$ Congresso BrMass.
WATERMAN, P. G. \& S. MOLE. 1994. Analysis of phenolic plant metabolites. Ecological methods and concepts (WILEY ed.) Vol. 1, pp. 238.

ZACCHINO, S. A. 2004. Productos naturales y análogos sintéticos con propiedades inhibitorias de hongos ftopatogénicos y oportunistas humanos. Estudios de mecanismos de acción. Rev. Soc. Quím. México 1: 48.

ZAMPINI I.C., N. CUDMANI \& M. I. ISLA. 2007. Actividad antimicrobiana de plantas medicinales argentinas sobre bacterias antibiótico-resistentes. Acta Bioquim. Clin. L. 4: 385-393.

ZULOAGA F. O., O. MORRONE \& M. J. BELGRANO (eds.). 2008. Catálogo de las plantas vasculares del Cono Sur (Argentina, Sur de Brasil, Chile, Paraguay y Uruguay). II. Monogr. Syst. Bot. Missouri Bot. Gard. 107: 1905-1908. Disponible en: http://www.darwin. edu.ar/ [Acceso: mayo 2012].

Recibido el 21 de junio de 2014, aceptado el 23 de septiembre de 2014. 
